\title{
Unified Expressions Of All Integral Variational Principles
}

\author{
Yong-Chang Huang ${ }^{1,4}$ Xi-Guo Lee ${ }^{2}$ M. X. Shao ${ }^{3}$ \\ ${ }^{1}$ Institute of Theoretical Physics, College of Applied Sciences, \\ Beijing University of Technology, Beijing 100022, P. R. China \\ ${ }^{2}$ Institute of Modern Physics, Chinese Academy of Sciences, \\ P.O.Box 31, Lanzhou, 730000, China \\ ${ }^{3}$ Department of Physics, Beijing Normal University, \\ Beijing 100875, P. R. China \\ ${ }^{4}$ CCAST (World Lab.), P. O. Box 8730, Beijing 100080, P. R. China \\ Key Words: Euler-Lagrange equation, variational principle, Noether theorem \\ PACS No.: 11.10.Ef, 11.30.-j \\ January 25, 2018

\begin{abstract}
In terms of the quantitative causal principle, this paper obtains a general variational principle, gives unified expressions of the general, Hamilton, Voss, Hölder, Maupertuis-Lagrange variational principles of integral style, the invariant quantities of the general, Voss, Hölder, Maupertuis-Lagrange variational principles are given, finally the Noether conservation charges of the general, Voss, Hölder, Maupertuis-Lagrange variational principles are deduced, and the intrinsic relations among the invariant quantities and the Noether conservation charges of the all integral variational principles are achieved.
\end{abstract}

\section{Introduction}

Physical laws may be expressed by variational principle not only by differential formulas, in fact, differential formulas can be derived from variational principle[1], and there are the variational principles of differential and integral styles[2,3]. Hilbert outlined 23 major mathematical problems to be studied in the coming century, variational problem is one of the problems. And Hilbert's address is still important and should be researched by anyone interested in pursuing research in mathematics and physics $[4,5]$.

The unified expressions of the all differential variational principles is presented by the quantitative causal principle (QCP) derived from the no-loss-no-gain principle in Ref.[6]. Using the no-loss-no-gain homeomorphic map transformation satisfying QCP, Ref.[7] solves the problem of the non-perfect properties of the Volterra process, gains the exact strain tensor formulas in condensed matter theory. QCP reflects that everything in the universe can not have cause without result or have result without cause, and any serious science is always quantitatively expressed by 
some equations, changes of some quantities in the equations must result in the changes of the other quantities in the equations so that the right hand sides of the equations keep no-loss-no-gain, namely, keep a kind of invariant symmetry (i.e., maintain zero invariant symmetry ), therefore QCP is general principle in physics, and QCP has the widest applications in both physics and mathematics. It can be seen what this paper is a development of Refs. $[4,5,6,8,9]$ 's researches about the relationships of causal principle, symmetry and variational principle. In statistical theory, statistical quantitative causal relations in expressions of equations are also satisfied, thus, definite results may be deduced[10].

In this paper, Sect. II gives the unified expressions of all integral variational principles, Sect. III shows up conservation quantities of all integral variational principles, Sect. IV represents Noether conservation charges of all integral variational principles, the last Sect. is summary and conclusions.

\section{Unified Expressions Of All Integral Variational Principles}

Ref.[6] rigorously gives the expression of QCP derived from the no-loss-no-gain principle. In fact, in physics, the quantitative actions (cause) of some quantities must lead to the corresponding equal effects (result), which is just a expression of the no-loss-no-gain principle $[6,7,8]$, therefore, we obtain[6]

Quantitative Causal Principle: In a general physical system, how much loses (cause), there must be, how much gains (result), or, quantitative actions (cause) of some quantities must result in the corresponding equal effects (result).

The principle, then, may be concretely expressed as

$$
D A-C A=0
$$

Eq.(2.1)'s physical meaning is that real physical result coming from any operator's set D acting on A must result in appearance of set $\mathrm{C}$ acting on A so that Eq.(2.1)'s right hand side seriously keeps no-loss-no-gain, namely, making DA equate to $\mathrm{CA}$, the whole process satisfies $\mathrm{QCP}$ with no-

loss-no-gain characteristic property. When A is a action, C is identity, and D is group operator of infinitesimal continuous transformation, the physical process is that the whole system with group D symmetry satisfies QCP, that is, the action A takes limit value under the transformation of group D

$$
\triangle A=A^{\prime}-A=\int_{t_{1}^{\prime}}^{t_{2}^{\prime}} L^{\prime}\left(q^{\prime}, \dot{q}^{\prime}, t^{\prime}\right) d t^{\prime}-\int_{t_{1}}^{t_{2}} L(q, \dot{q}, t) d t=0
$$


where $D A=A^{\prime}$, and the general infinitesimal transformations are[11]

$$
t^{\prime}=t+\triangle t, q_{i}{ }^{\prime(r)}=q_{i}{ }^{(r)}+\triangle q_{i}^{(r)},(r=0,1)
$$

Not losing generality, let us define

$$
L^{\prime}\left(q^{\prime}, \dot{q}^{\prime}, t^{\prime}\right)=L\left(q^{\prime}, \dot{q}^{\prime}, t^{\prime}\right)+f\left(q^{\prime}, \dot{q}^{\prime}, t^{\prime}\right)
$$

where $\mathrm{f}$ is a smooth function. It, thus, follows that

$$
\triangle A=\int_{t_{1}}^{t_{2}}\left[f\left(q^{\prime}, \dot{q}^{\prime}, t^{\prime}\right)+\triangle(f+L)+(f+L) \frac{d \Delta t}{d t}\right] d t
$$

Making Eq.(2.5) in order, we have

$\triangle A=\int_{t_{1}}^{t_{2}}\left\{f(q,: q, t)+\sum i \sum\left[\frac{\partial(L+f)}{\partial q_{i}}-\frac{d}{d t} \frac{\partial(L+f)}{\partial \cdot q_{i}}\right] \delta q_{i}+\frac{d}{d t}\left[\sum i \sum \frac{\partial(L+f)}{\partial{ }^{\prime} q_{i}} \delta q+(L+f) \Delta t\right]\right\} d t$

Since Eq.(2.4) is a limit process, not losing generality, neglecting two-order infinitesimal quantity, it follows that

$$
f=f_{0}+\varepsilon_{\sigma} \frac{d g^{\sigma}}{d t}, \sigma=1,2, \cdots, m
$$

where $\varepsilon_{\sigma}(\sigma=1,2, \cdots, m)$ are linearly independent infinitesimal parameters of Lie group D.

Substituting Eq.(2.7) into Eq.(2.6), neglecting two order infinitesimal quantity, then Eq.(2.6) is simplified as

$\triangle A=\int_{t_{1}}^{t_{2}}\left\{f_{0}(q,: q, t)+\sum i \sum\left[\frac{\partial\left(L+f_{0}\right)}{\partial q_{i}}-\frac{d}{d t} \frac{\partial\left(L+f_{0}\right)}{\partial \cdot q_{i}}\right] \delta q_{i}+\frac{d}{d t}\left[\sum i \sum \frac{\partial\left(L+f_{0}\right)}{\partial \cdot q_{i}} \delta q_{i}+\left(L+f_{0}\right) \Delta t+g\right]\right\} d t$

in which $\mathrm{g}=\varepsilon_{\sigma} g^{\sigma}, \sigma=1,2, \cdots, m$. For the third term in Eq.(2.8) taking the endpoint condition as zero, because $f_{0}(q,: q, t)$ does not contain one order infinitesimal quantity, and using $\triangle A=0$ and the arbitration of one order infinitesimal quantity $\delta q_{i}$, we obtain $\mathrm{f}_{0}=0$, Eq.(2.8) is thus simplified as

$$
\triangle A=\int_{t_{1}}^{t_{2}} \sum i\left\{\sum\left[\frac{\partial L}{\partial q_{i}}-\frac{d}{d t} \frac{\partial L}{\partial \cdot q_{i}}\right] \delta q_{i}+\frac{d}{d t} \sum i\left(\sum \frac{\partial L}{\partial \cdot q_{i}} \delta q_{i}+L \Delta t+g\right)\right\} d t
$$

In the past, $\mathrm{f}_{0}=0$ is well known widely to be the result keeping Euler-Lagrange Equation invariant, now it is seen that under the condition of Eq.(2.7), $\mathrm{f}_{0}=0$ is not the result that maintains 
Euler-Lagrange equation invariant, which is just the result satisfying QCP. This is the new result that has not been obtained in the past.

Using Eq.(2.9) may give the unified expressions and the intrinsic relations of all variational principles of integral style. In terms of the requirement that integrating the second term in Eq.(2.9) vanishes, it follows that the conditions of the new general variational principle are: for the fixed $\mathrm{t}_{1}$ and $\mathrm{t}_{2},\left.\delta q_{i}\right|_{t=t_{1}}=\left.\delta q_{i}\right|_{t=t_{2}}=0,\left.\Delta t\right|_{t=t_{1}}=\left.\Delta t\right|_{t=t_{2}}=0$ and $\mathrm{g}\left(\mathrm{t}_{1}\right)=\mathrm{g}\left(\mathrm{t}_{2}\right)$, and using arbitration of $\delta q_{i}$, we obtain Euler-Lagrange Equation

$$
\frac{\partial L}{\partial q_{i}}-\frac{d}{d t} \frac{\partial L}{\partial \cdot q_{i}}=0
$$

When taking equal time $\Delta \mathrm{t}=0$ in Eq.(2.9), we obtain Hamilton variational principle

$$
\delta A=\int_{t_{1}}^{t_{2}}\left\{\frac{d}{d t}\left[\sum i \sum \frac{\partial L}{\partial: q_{i}} \delta q_{i}+g\right]+\sum i \sum\left[\frac{\partial L}{\partial q_{i}}-\frac{d}{d t} \frac{\partial L}{\partial: q_{i}}\right] \delta q_{i}\right\} d t
$$

Using the requirement that integrating the first term in Eq.(2.11) is zero, it follows that the conditions of Hamilton variational principle are: for the fixed $t_{1}$ and $t_{2},\left.\delta q_{i}\right|_{t=t_{1}}=\left.\delta q_{i}\right|_{t=t_{2}}=0$ and $\mathrm{g}\left(\mathrm{t}_{1}\right)=\mathrm{g}\left(\mathrm{t}_{2}\right)$, and looking from $\mathrm{E}^{q}$ space, which is fixed endpoint variational. Using arbitration of $\delta q_{i}$,we get Euler-Lagrange Equation (2.10). Due to

$$
\triangle q_{i}^{(r)}=\delta q_{i}^{(r)}\left(t^{\prime}\right)+q_{i}^{(r+1)} \triangle t, r=0,1
$$

Eq.(2.9) can be rewritten as

$\triangle A=\int_{t_{1}}^{t_{2}}\left\{\sum i \sum\left(\frac{\partial L}{\partial q_{i}}-\frac{d}{d t} \frac{\partial L}{\partial \cdot q_{i}}\right)\left(\triangle_{p} q_{i}-: q_{i} \triangle t\right)+\frac{d}{d t}\left[\sum i \sum\left(\frac{\partial L}{\partial \cdot q_{i}}\right)\left(\triangle_{p} q_{i}-: q_{i} \triangle t\right)+L \triangle t+g\right]\right\} d t$

Because the requirement that integrating the second term in Eq.(2.13) vanishes, we obtain that the conditions of Voss variational principle are: unequal time variational, $\left.\Delta t\right|_{t=t_{1}}=\left.\Delta t\right|_{t=t_{2}}=$ $0,\left.\triangle_{p} q_{i}\right|_{t=t_{1}}=\left.\triangle_{p} q_{i}\right|_{t=t_{2}}=0$ and $\mathrm{g}\left(\mathrm{t}_{1}\right)=\mathrm{g}\left(\mathrm{t}_{2}\right)$. Because $\triangle_{p} q_{i}-: q_{i} \triangle t(i=1,2, \cdots, n$. $)$ construct virtual variational of the system, we similarly get the Euler-Lagrange Equation (10) from Eq.(2.13). When inserting Eq.(2.12) into Eq.(2.9), and using

$$
\begin{gathered}
\frac{d}{d t}\left(\sum i \sum \frac{\partial L}{\partial \cdot q_{i}} \cdot q_{i}-L\right)=-\sum i \sum\left(\frac{\partial L}{\partial q_{i}}-\frac{d}{d t} \frac{\partial L}{\partial \cdot q_{i}}\right): q_{i}-\frac{\partial L}{\partial t} \\
\triangle A=\int_{t_{1}}^{t_{2}}\left(\triangle L+L \frac{d \triangle t}{d t}+\frac{d g}{d t}\right) d t
\end{gathered}
$$


we further make Eq.(2.9) in order, it follows that

$\int_{t_{1}}^{t_{2}}\left\{\triangle L-\sum i \sum\left(\frac{\partial L}{\partial q_{i}}-\frac{d}{d t} \frac{\partial L}{\partial \cdot q_{i}}\right) \triangle q_{i}+\sum i \sum \frac{\partial L}{\partial \cdot q_{i}}: q_{i} \frac{d \triangle t}{d t}-\frac{\partial L}{\partial t} \triangle t\right\} d t=\int_{t_{1}}^{t_{2}} \sum i \sum \frac{d}{d t}\left[\left(\frac{\partial L}{\partial \cdot q_{i}}\right) \triangle q_{i}\right] d t$

Using the demand that keeps Eq.(2.16)'s right hand side no-loss-no-gain to equate zero, we obtain that the conditions of Hölder variational principle are: unequal time variational, $\triangle \mathrm{t}$ may be not to equate to zero and $\left.\triangle q_{i}\right|_{t=t_{1}}=\left.\Delta q_{i}\right|_{t=t_{2}}=0$.

When the system satisfies the Euler-Lagrange Eq.(2.10), we get the simplified expression of Eq.(2.16) of Hölder variational principle as follows

$$
\int_{t_{1}}^{t_{2}}\left\{\triangle L+\sum i \sum\left[\frac{\partial L}{\partial: q_{i}}: q_{i}\right] \frac{d \triangle t}{d t}-\frac{\partial L}{\partial t} \triangle t\right\} d t=0
$$

It is seen that Hölder variational principle is originated from Eq.(2.9) satisfying QCP yet, looking in $\mathrm{E}^{q}$ space, the variational is the variational of variant endpoint along time $\mathrm{t}$ axis.

When taking equal time variational in Eq.(2.17), it is simplified as Hamilton variational principle[12]

$$
\delta A=\int_{t_{1}}^{t_{2}} \delta L d t=0
$$

It is looked that Hamilton variational principle not containing $\mathrm{g}$ is yet a simplified expression of Hölder variational principle under the condition of equal time variational. In expression (2.17) of Hölder variational principle, as $\mathrm{L}=\mathrm{L}(q,: q)$, one has $\partial L / \partial t=0$. When there exists motion trajectory integration[12]

$$
H=T+V=\sum i \sum \frac{\partial L}{\partial \cdot q_{i}}: q_{i}-L=\text { const }
$$

it follows from Eq.(2.19) that

$$
\triangle H=\triangle \sum i \sum\left(\frac{\partial L}{\partial: q_{i}}: q_{i}\right)-\triangle L=0
$$

Substituting $\partial L / \partial t=0$ and Eq.(20) into Eq.(2.17), we have

$$
\int_{t_{1}}^{t_{2}}\left(\triangle(2 T)+2 T \frac{d \triangle t}{d t}\right) d t=\triangle \int_{t_{1}}^{t_{2}}(2 T) d t=0
$$

where $\sum i \sum\left(\partial L / \partial: q_{i}\right) q_{i}=2 T$ has been used. 
Eq.(21) is just the expression of Maupertuis-Lagrange variational principle. It is watched that the Maupertuis-Lagrange principle's conditions is Hölder's, i.e, the endpoints are motional[3]. Therefore, the unified expressions of the all variational principles of integral style is shown by means of QCP, and the other high order principles etc can be analogously discussed.

\section{Conservation Quantities of All Integral Variational Prin- ciples}

Now we discuss their conservation quantities about the above all principles.

Taking endpoint condition $(\cdots)\left(t_{1}\right)=(\cdots)\left(t_{2}\right)$ in Eq.(2.9), when using arbitrations of $t_{1}$, $\mathrm{t}_{2}$ and $\mathrm{t}$ in given $\left[\mathrm{t}_{01}, \mathrm{t}_{02}\right]$ and $\delta q_{i}$, we obtain Euler-Lagrange Eq.(2.10) and the following general conservation quantity of the general variational principle

$$
\sum i \sum \frac{\partial L}{\partial: q_{i}} \delta q_{i}+L \triangle t+g=\text { const }
$$

Analogous to the discussions of Eq.(3.22), the conservation quantity of Voss variational principle may be obtained. On the other hand, because Eq.(3.22) is the general conservation quantity, the conservation quantity of Voss variational principle can also be achieved by substituting $\delta q_{i}=$ $\triangle_{p} q_{i}-: q_{i} \triangle t$ into the general conservation expression (3.22) as follows

$$
\sum i \sum \frac{\partial L}{\partial \cdot q_{i}}\left(\triangle_{p} q_{i}-: q_{i} \triangle t\right)+L \triangle t+g=\text { const }
$$

The general integral conservation quantity Eq.(3.22) is compared with the differential conservation quantity[6]

$$
\sum i \sum \frac{\partial L}{\partial \cdot q_{i}} \delta q_{i}+L \triangle t=\text { const }, \sigma=1,2, \cdots, m
$$

$\mathrm{g}$ is the more part than the differential style's. When taking equal time variational in the general conservation expression (3.22), we obtain conservation quantity of Hamilton variational principle

$$
\sum i \sum \frac{\partial L}{\partial \cdot q_{i}} \delta q_{i}+g=\text { const }
$$

In the similar reason, it follows from Eq.(2.16) that the conservation quantity of Hölder variational principle

$$
\sum i \sum \frac{\partial L}{\partial \cdot q_{i}} \triangle q_{i}=\text { const }
$$


Since the expression (2.21) of Maupertuis-Lagrange variational principle is just the result of Hölder variational principle under the constraint conditions (2.19) and $\partial L / \partial t=0$, thus the corresponding conservation quantity is still Eq.(3.26). Substituting $L=2 T-$ const. into Eq.(3.26) we further deduces the conservation quantity of Maupertuis-Lagrange variational principle as follows

$$
\sum i \sum \frac{\partial 2 T}{\partial \cdot q_{i}} \triangle q_{i}=\text { const }
$$

It is well known that it is usually neglected that the invariant quantities of Voss, Hölder, Maupertuis-Lagrange variational principles, thus, the invariant quantities of the new general, Voss, Hölder, Maupertuis-Lagrange variational principles are first given, then conservation quantities of all integral variational principles are first deduced, and the intrinsic relations among the invariant quantities of the all integral variational principles are first found.

\section{Noether Conservation Charges of All Integral Variational Principles}

Now we use the conservation quantities above to find the corresponding Noether conservation charges of the systems with invariant properties of Lie group $\mathrm{D}_{m}$. Let's consider the infinitesimal transformations of the spacetime coordinates by Lie group $\mathrm{D}_{m}$ as follows[11, 13]

$$
\begin{gathered}
t^{\prime}=t^{\prime}(q(t),: q(t), t, \alpha) \doteq t+\Delta t=t+\varepsilon_{\sigma} \tau^{\sigma}(\sigma=1,2, \cdots, m) \\
q_{i}^{\prime(r)}=q_{i}^{\prime(r)}(q(t),: q(t), t, \alpha) \doteq q_{i}^{(r)}+\Delta q_{i}^{(r)}=q_{i}^{(r)}+\varepsilon_{\sigma}\left(\xi_{i}^{\sigma}\right)^{(r)},(r=0,1)
\end{gathered}
$$

where $\varepsilon_{\sigma}(\sigma=1,2, \cdots, m)$ are infinitesimal parameters corresponding to $\alpha_{\sigma}, \alpha_{\sigma}(\sigma=1,2, \cdots, m)$ are $m$ linearly independent infinitesimal continuous transformation parameters of Lie group $\mathrm{D}_{m}$, $\tau^{\sigma}$ and $\left(\xi_{i}^{\sigma}\right)^{(r)}$ are the infinitesimal generated functions as follows

$$
\begin{gathered}
\tau^{\sigma}=\left.\frac{\partial t^{\prime}(q(t),: q(t), t, \alpha)}{\partial \alpha_{\sigma}}\right|_{\alpha=0}(\sigma=1,2, \cdots, m) \\
\left(\xi_{i}^{\sigma}\right)^{(r)}=\left.\frac{\partial q_{i}^{\prime(r)}(q(t), . q(t), t, \alpha)}{\partial \alpha_{\sigma}}\right|_{\alpha=0}(\sigma=1,2, \cdots, m ; r=0,1)
\end{gathered}
$$

Using Eqs.(2.7), (2.12), (3.22), (4.28-31) and $\varepsilon_{\sigma}$ 's arbitration, we obtain the Noether conservation charges of the general variational principles as follows

$$
\sum i\left\{\sum \frac{\partial L}{\partial: q_{i}}\left(\left(\xi_{i}^{\sigma}\right)-: q_{i} \tau^{\sigma}\right)+L \tau^{\sigma}+g^{\sigma}\right\}(t)=\text { const. }(\sigma=1,2, \cdots, m)
$$


Making use of Eqs.(2.7), (2.12), (3.23), (4.28-31) and $\varepsilon_{\sigma}$ 's arbitration, we get the Noether conservation charges of Voss variational principles is still Eq.(3.23) but substituting $\left(\xi_{i}^{\sigma}\right)$ with $\left(\xi_{i}^{\sigma}\right)_{p}$.

Using Eqs.(2.7), (2.12), (3.25), (4.28-31) and $\varepsilon_{\sigma}$ 's arbitration or in the general Eq.(32) let $\Delta t=\tau^{\sigma}=0$, we obtain Noether conservation charges of Hamilton variational principle

$$
\sum i \sum \frac{\partial L}{\partial \cdot q_{i}}\left(\xi_{i}^{\sigma}\right)(t)+g(t)^{\sigma}=\text { const. }(\sigma=1,2, \cdots, m)
$$

Utilizing $\varepsilon_{\sigma}$ 's arbitration or in the general Eq.(4.32) let $\mathrm{g}=0$ and $\Delta t=\tau^{\sigma}=0$, we obtain Noether conservation charges of Hölder variational principle as follows

$$
\sum i \sum \frac{\partial L}{\partial \cdot q_{i}}\left(\xi_{i}^{\sigma}\right)(t)=\text { const. }(\sigma=1,2, \cdots, m)
$$

Let $L=2 T$ - const. , we can obtain Noether conservation charges of Maupertuis-Lagrange variational principle

$$
\sum i \sum \frac{\partial 2 T}{\partial: q_{i}}\left(\xi_{i}^{\sigma}\right)(t)=\text { const. }(\sigma=1,2, \cdots, m)
$$

We, thus, get the conclusion that Noether conservation charge of the general variational principle is general, and the Noether conservation charges of the other variational principles are the special examples of the general variational principle under different conditions. And it can be seen from the above researches that all variational principles of integral style deduce the same Euler-Lagrange Eq.(2.10), and there may be the different conservation quantities of the different systems, The former characterizes that physical laws don't depend on different conditions, the latter characterizes that physical manifestations of systems may be many kinds of variations, because the invariant quantities are relative to the physical observable quantities. Since the quantitative causal principle is more general, its applications to high order Lagranges etc will be written in the other papers.

\section{Summary and Conclusion}

In terms of the mathematical expression of $\mathrm{QCP}$, this paper gives a general variational principle, shows unified expressions of the general, Hamilton, Voss, Hölder, Maupertuis-Lagrange variational principles of integral style, finds the intrinsic relations among the different integral variational principles, it is shown that under the condition of Eq.(2.7), $\mathrm{f}_{0}=0$ is not the result that maintains Euler-Lagrange equation invariant, which is just the result satisfying QCP. The invariant quantities 
of the general, Voss, Hölder, Maupertuis-Lagrange variational principles are given, and the intrinsic relations among the invariant quantities and the Noether conservation charges of the all integral variational principles are found. In fact, the above discussions and Ref.[6] make the expressions of the past scrappy numerous variational principles be unified into the relative consistent system of the all variational principles in terms of $\mathrm{QCP}$, which is essential for researching the intrinsic relations among the past scrappy numerous variational principles and their Noether theorems and for further making their logic simplification and clearness.

ACKNOWLEDGMENTS:

This work was supported by Chinese Academy of Sciences Knowledge Innovation Project (KJCX2-SW-No2), National Natural Science Foundations of China $(10435080,10575123)$.

The authors are grateful to Prof. Z. P. Li for useful discussion.

\section{References}

[1] E. A. Desloge, Classical Mechanics, Vol. I, II, John Wiley and Sons, New York, (1982).

[2] H. Baruh, Analytical dynamics, Monograph Boston, MA : WCB/McGraw-Hill, 1999.

[3] L. A. Pars, A Treatise on Analytical Dynamics, Heinemann Educational Books Ltd, London, (1965).

[4] Ivor Grattan-Guinness, A sideways look at Hilbert's twenty-three problems of 1900, Notices of the AMS, 47(2000)752.

[5] F. Browder (ed.), Mathematical Developments Arising from Hilbert's Problems, Symposia in Pure Mathematics, Amer. Math. Soc. 28, Providence, Rhode Island, 1976.

[6] Y. C. Huang, Mechanics Research Communications, 30 (2003)567.

[7] Y. C. Huang and B. L. Lin, Physics Letters, A299 (2002) 644; ibid, A306 (2003)369.

[8] Y. C. Huang, F. C. Ma and N. Zhang, Modern Physics Letters, B18 (2004) 1367.

[9] P. Curie, Journal de physique, (Paris), 3rd series, 3(1894)395.

[10] Y. C. Huang, P. C. Zou and Q. Gao, Archive of Applied Mechanics, 67(1997)151.

[11] D. S. Djukic, Int. J. Non-linear Mech., 8(1993)479.

[12] R. M. Rosenberg, Analytical Dynamics of Discrete System, Plenum, New York (1977). 
[13] Z. P. Li, Int. J. Theor. Phys., 26(1987)853. 